\title{
The Relationship between Professional Identity and Career Maturity among Pre-Service Kindergarten Teachers: The Mediating Effect of Learning Engagement
}

\author{
Liying Zhang ${ }^{1}$, Meirong Chen ${ }^{2}$, Xiaoqing Zeng ${ }^{*}$, Xinqiang Wang3 \\ ${ }^{1}$ School of Education, Jiangxi Normal University, Nanchang, China \\ ${ }^{2}$ Department of Education, Nanchang Normal University, Nanchang, China \\ ${ }^{3}$ School of Psychology, Jiangxi Normal University, Nanchang, China \\ Email: *zxq3701@163.com
}

How to cite this paper: Zhang, L.Y., Chen, M.R., Zeng, X.Q. and Wang, X.Q. (2018) The Relationship between Professional Identity and Career Maturity among Pre-Service Kindergarten Teachers: The Mediating Effect of Learning Engagement. Open Journal of Social Sciences, 6, 167-186.

https://doi.org/10.4236/jss.2018.66016

Received: May 17, 2018

Accepted: June 17, 2018

Published: June 20, 2018

Copyright (c) 2018 by authors and Scientific Research Publishing Inc. This work is licensed under the Creative Commons Attribution International License (CC BY 4.0).

http://creativecommons.org/licenses/by/4.0/

(c) (i) Open Access

\begin{abstract}
Objective: To investigate the mediating role of leaning engagement in the relationship between professional identity and career maturity. Methods: 711 pre-service kindergarten teachers completed the Professional Identification Scale, the College Students' Career Maturity Questionnaire and the Utrecht Work Engagement Scale-student. Results: 1) There are significant difference in professional identity and career maturity on grades, different level of parents' education and whether part-time work. 2) Professional identity, career maturity, and learning engagement were significantly correlated with each other. 3) Career maturity partially mediated the relationship between professional identity and learning engagement. Conclusion: Professional identity directly influences learning engagement, and also influences learning engagement through the partial mediating effects of career maturity.
\end{abstract}

\section{Keywords}

Pre-Service Kindergarten Teachers, Professional Identity, Leaning

Engagement, Career Maturity

\section{Introduction}

The National Mid and Long-Term Education Reform and Development Plan (2010-2020) proposes to popularize pre-primary education and reach a $95 \%$ gross enrolment rate by 2020. In order to accommodate more children, kindergartens are rapidly expanding and desire for kindergarten teachers. As the 
orientation of employment for preschool education major mainly focuses on children, the employment prospect shall be preferable. However, It's seems not optimistic for preschool education major in recent years according to the current status. There are several issues exist such as the low share of graduates, lower salary of graduates, and low professional ability etc. Lu (2016), when investigating the employment situation of pre-service kindergarten teachers in 2013 and 2012, found that the employment rate in the two years reached $100 \%$ [1]. Of course, among them, very few students choose to be self-employed. But when it comes to 2014, it was found that the employment rate was relatively low. Xu (2017) believes that in the context of the National Qualification Examination for Teacher Qualifications, the employment of preschool teachers and students is facing challenges, such as perplexed, poor job prospect and increasing pressure [2]. Career maturity refers to the level of career development corresponding to the individual's career development stage (Crites \& Savickas, 1996) [3]. It is not only an essential evaluation index of the level of career development, but also one of the key factors to assess the success of college students in employment. Preschool normal students are the main source of preschool teachers in our country. Their professional attitude and ability are directly related to their teaching literacy in the future and influence the development of preschool education. Moreover, less study is interested in the professional maturity of preschool education teachers. Therefore, it is extremely necessary to conduct in-depth research on the influencing factors of preschool teacher's career maturity so as to lay the foundation for subsequent effective intervention.

The Professional identity of teachers is an important part of teachers' professional psychological quality, which determines the degree of subjective effort and attitude toward education development (Wei \& Song, 2005) [4]. The professional identity for normal students refers to the comprehensive acceptance and recognition of teachers' occupations and the current status, which include professional efficacy, professional values, professional intention and expectations, and professional volition (Wang, Zeng, Zhang \& Li, 2010) [5]. Crites (1995) [6] believes that career maturity refers to the level of development of individuals in a given set of career-related development tasks. Savickas (1994) [7] holds that career maturity is an individual's readiness to make appropriate career decisions based on certain information and manage their career development tasks. Liu (2009) [8] defines the degree of career maturity of college students as "the degree to which a college student can make career decisions that are compatible with his or her career development stage and prepare for the corresponding career goals, which includes career decision-making knowledge and career decision-making attitudes". Studies have shown that professional identity and professional maturity are closely related. Professional identity is one of the most important factors affecting professional success and satisfaction (Vilija Stanišauskienė \& Gintarè Edintaitė, 2016) [9]. Professional identity and professional maturity have similar formation mechanisms: they are based on the organic integration of the individual with social environment, self-concept and the 
real world. Students who have a better understanding of the status of normal students and their teaching profession are more willing to devote time and efforts to the study of professional knowledge and teacher skills training. Related studies have shown that there is a significant positive correlation between teachers' professional identity and Career maturity. The former has a good predictive power to the latter, that is, the higher the teacher's professional identity (non-extreme state), the higher the professional maturity and vice versa (Hou, Hu \& Guo, 2017) [10]. In addition, professional identity also associates with social support, learning motivation, career resilience, and occupational mental health (Wang \& Zhang, 2012) [11]. These factors have positive effects on contributing to the formation of occupational maturity. (Ma, Wei \& Liu, 2013) [12]. Therefore, this study assumes that professional identity has a positive predictive effect on career maturity.

The study of study engagement began from research for time on task in the United States in the 1930s. Connell (1990) [13] believes that study engagement includes three aspects: behavior, emotion and cognition. Schaufeli (2002) [14] divides study engagement into three dimensions, which is vitality, dedication, and concentration, and believes that study engagement is a state of long-term stability. Study engagement is a lasting, positive emotional and cognitive psychological state of mind related to learning, research, and employment. For normal university students, study engagement refers to their integration of behavioral, emotional, and cognitive in the course of study, professional practice, and extracurricular activities. Yang (2017) [15] believes there are significant positive correlations among professional identity, various dimensions, and study engagement. The Career value and professional efficacy are effective predictor variable to vigor, dedication, and concentration. An empirical study by Tan \& Liu (2014) [16] shows that there are significant correlations between teacher professional identity's factors and study engagement's factors. Such professional factors as professional efficacy, professional volition which identified by normal students and teachers can effectively predict the vitality, dedication and concentration of study engagement. Wang (2015) [17] believes that there are significant positive correlations between the three dimensions of teachers' professional identity and their study engagement and academic achievement. It can be concluded that there is a significant positive correlation between teacher professional identification and study engagement and professional identity is an effective predictor of study engagement. Research by relevant scholars also shows that study engagement is significantly positive in predicting career maturity. Zhao (2013) [18] pointed out that there is a significant correlation between the vitality, dedication dimension of study engagement and the overall career maturity of teachers, ability maturity, attitude maturity, and the inherent orientation of their dimensions. There is also a significant correlation between maturity and intrinsic orientation. Tian (2016) [19] believes that there is a significant positive correlation between study engagement and teacher career maturity $(r=0.321, p<$ 
0.01). The level of time management of normal students not only directly affects the degree of career maturity, but also can improve them indirectly, achieve the development of self-value. They are willing to make efforts and invest more energy in the process of career preparation which let them continuous growth and have a further recognize career maturity of normal students. Zhang (2017) [20] believes that study engagement has a significant positive effect on the employment ability of college students, and that the increase in behavior, cognition, and emotional engagement is conducive to improving the professional development ability of college students. Therefore, it can be inferred that the study engagement plays an intermediary role between teachers' professional identity and career maturity.

In conclusion, this study incorporated the study engagement into the intermediary model to further analyze the effect of teachers' professional identity on career maturity of normal student

\section{Methodology}

The study was aimed to investigate the mediating role of leaning engagement in the relationship between professional identity and career maturity. Quantitative data were collected through survey questionnaires distributed to members of pre-service kindergarten teachers from two topical normal universities in Nanchang, Jiangxi Province. For the diversity of respondents and efficient investigation, we choose the two institutions as survey sites, using the Professional Identification Scale, the College Students' Career Maturity Questionnaire and the Utrecht Work Engagement Scale-student to investigate.

\subsection{Sample Population}

Sample Population members of the pre-service kindergarten teachers from two topical normal universities in Nanchang, Jiangxi Province. 765 questionnaires were distributed this time, and 711 valid questionnaires were obtained. The effective recovery rate was 92.94\%. Among them are 24 boys and 687 girls; 377 specialists and 334 undergraduates; 410 students from cities and 301 from rural areas. The age ranged from 14 to 25 years and the average age was $19.01(\mathrm{SD}=$ $0.36)$.

\subsection{The Instrument Used}

\subsubsection{Professional Identity Inventory for Normal Student}

The professional identity for normal students refers to the comprehensive acceptance and recognition of teachers' occupations and the current status (Wang, Zeng, Zhang \& Li, 2010) [5]. In this study, the professional identity inventory for normal school students prepared by Wang et al. (2010) [5] are being used. The inventory is composed of 12 items, which consists of four dimensions: professional intention and expectations, professional volition, professional value, and professional efficacy with 3 items in each dimension (Wang, Zhu, Liu, \& Chen, 
2017) [21]. The 5-point scoring method was used, and subjects were asked to score according to their own actual situation. Due to the special nature of preschool education normal student, the title of the inventory is modified. For example, "teacher" is changed to "kindergarten teacher", "student" is changed to "normal university student". " 1 " means "completely incompatible"; " 5 " means "fully compliant". In this study, the Cronbach $\alpha$ coefficient of the inventory was 0.84 .

\subsubsection{Study Engagement Inventory}

Study engagement is a lasting, positive emotional and cognitive psychological state of mind related to learning, research and employment. Study engagement into three dimensions, which is vitality, dedication, and concentration (Fang, Shi, \& Zhang, 2008) [22]. The Chinese version of study engagement revised by Fang et al. (2008) [22] according to the study engagement compiled by Schaufeli (2002) [14], etc. is being used. The inventory consists of 17 items, which can be divided into three dimensions: vitality (6 items), dedication (5 items) and concentration (6 items). Richter's 7 points method is being used in this inventory. "0" for "never"; " 6 " for "always". The higher the score, the more study engaged. In this study, the Cronbach $\alpha$ coefficient of the inventory was 0.94 .

\subsubsection{Career Maturity Inventory for College Students}

Liu (2009) [8] defines the degree of career maturity of college students as "the degree to which a college student can make career decisions that are compatible with his or her career development stage and prepare for the corresponding career goals, which includes career decision-making knowledge and career decision-making attitudes". The career maturity inventory for college students compiled by Liu (2009) [8] is included. This inventory contains 29 items which consist of 9 dimensions: professional self-knowledge, professional world knowledge, professional knowledge, strategic knowledge of interpersonal communication, initiative, independence, reality, utilitarianism and self-confidence. The 5-point scoring method of A Likter was used. In this study, the Cronbach $\alpha$ coefficient of the inventory was 0.73 .

\section{Findings}

\section{1. Pre-Service Kindergarten Teachers' Career Maturity, Whether They Have Part-Time Work Experience and Parents' Educational Level}

\subsubsection{Grade Difference of Vocational Maturity of Pre-Service Kindergarten Teachers}

The results showed that there were significant grade differences in professional knowledge, career decision making knowledge and career maturity among preschool normal school students. The difference was reflected in grade $4>$ grade $3>$ prep $>$ grade $2>$ grade 1 . See Table 1 . 
Table 1. Grade differences in career maturity.

\begin{tabular}{|c|c|c|c|c|}
\hline Variables & Grade & $M \pm S D$ & $F$ & $L S D$ \\
\hline \multirow[t]{7}{*}{ Professional knowledge } & 0 & $3.21 \pm 0.48$ & & $1<4^{* *}$ \\
\hline & 1 & $3.09 \pm 0.44$ & & $2<4^{\star *}$ \\
\hline & 2 & $3.09 \pm 0.44$ & & 4.21 \\
\hline & 3 & $3.20 \pm 0.39$ & & \\
\hline & 4 & $3.33 \pm 0.50$ & & \\
\hline & 0 & $3.43 \pm 0.49$ & & $1<3^{*}$ \\
\hline & 1 & $3.37 \pm 0.44$ & & $1<4^{* *}$ \\
\hline \multirow{7}{*}{$\begin{array}{c}\text { Professional } \\
\text { decision-making knowledge }\end{array}$} & 2 & $3.41 \pm 0.45$ & 3.17 & $2<4^{*}$ \\
\hline & 3 & $3.49 \pm 0.40$ & & \\
\hline & 4 & $3.60 \pm 0.44$ & & \\
\hline & 0 & $3.25 \pm 0.34$ & & $1<3^{*}$ \\
\hline & 1 & $3.18 \pm 0.30$ & & $1<4^{* *}$ \\
\hline & 2 & $3.21 \pm 0.29$ & 3.74 & $2<4^{* *}$ \\
\hline & 3 & $3.27 \pm 0.24$ & & \\
\hline Career maturity & 4 & $3.33 \pm 0.34$ & & \\
\hline
\end{tabular}

Note: ${ }^{*} \mathrm{p}<0.05,{ }^{* *} \mathrm{p}<0.01,{ }^{* * *} \mathrm{p}<0.001$. (same below) $0=$ preparatory course, $1=$ First grade, $2=$ Second grade, $3=$ Third grade, $4=$ Fourth grade.

\subsubsection{Occupational Maturity Difference between Pre-Service Kindergarten Teachers with Part-Time Job Experience and Those without Any Experience}

The results show that there were significant differences existing in normal school students with or without part-time work experience in aspects of professional self-knowledge, interpersonal communication strategy, initiative, self-confidence, reality, the knowledge and attitude of career decision-making and career maturity $(\mathrm{p}<0.05)$. The difference is that there are part-time and professional-related $>$ part-time but not professional-related $>$ no-part-time. See Table 2 .

\subsubsection{The Difference of Vocational Maturity of Pre-Service Kindergarten Teachers with Different Parental Education Level}

The results showed that there were significant differences in professional self-knowledge, interpersonal communication strategy, independence, self-confidence, career decision making knowledge and career maturity among pre-service kindergarten teachers with different father's education level $(\mathrm{p}<0.05)$. There were significant differences in professional self-knowledge, interpersonal communication strategy, independence, reality, utilitarianism, career decision making knowledge and career maturity among pre-service kindergarten teachers with different mothers' education level $(\mathrm{p}<0.05)$. The general difference is university and above $>$ secondary/secondary school $>$ primary school below. See Table 3 , Table 4. 
Table 2. Differences in career maturity of college students with or without part-time work experience.

\begin{tabular}{ccccc}
\hline Variables & Doing part-time job or not & $\mathrm{M} \pm \mathrm{SD}$ & $\mathrm{F}$ & LSD \\
\hline Professional self-knowledge & 1 & $3.55 \pm 0.60$ & & $1<3^{*}$ \\
& 2 & $3.50 \pm 0.55$ & 4.12 & \\
Interpersonal communication & 3 & $3.75 \pm 0.49$ & & \\
Strategy & 1 & $3.24 \pm 0.64$ & & $1<3^{* * *}$ \\
& 2 & $3.26 \pm 0.70$ & 8.27 & \\
Initiative & 3 & $3.60 \pm 0.64$ & & $1<3^{* * *}$ \\
& 1 & $3.45 \pm 0.68$ & & \\
& 2 & $3.51 \pm 0.64$ & 8.50 & \\
Self-confidence & 3 & $3.82 \pm 0.50$ & & \\
& 1 & $2.82 \pm 0.82$ & & $1<2^{* *}$ \\
& 2 & $3.05 \pm 0.80$ & 6.15 & \\
\hline
\end{tabular}

Note: 1 = No part-time, 2 = part-time but not profession-related, 3 = part-time and profession-related.

Table 3. Differences in career maturity of college students with different levels of father education.

\begin{tabular}{|c|c|c|c|c|}
\hline Variables & Father's educational level & $M \pm S D$ & $F$ & $L S D$ \\
\hline Professional & 1 & $3.75 \pm 0.60$ & & $1>2^{\star *}$ \\
\hline \multirow[t]{2}{*}{ Self-knowledge } & 2 & $3.56 \pm 0.56$ & 8.38 & \\
\hline & 3 & $3.44 \pm 0.58$ & & \\
\hline Interpersonal communication & 1 & $3.42 \pm 0.72$ & & $1>3^{\star *}$ \\
\hline \multirow[t]{2}{*}{ Strategy } & 2 & $3.28 \pm 0.65$ & 3.60 & \\
\hline & 3 & $3.18 \pm 0.67$ & & \\
\hline \multirow[t]{3}{*}{ Independence } & 1 & $2.67 \pm 0.65$ & & $1>3^{\star *}$ \\
\hline & 2 & $2.63 \pm 0.55$ & 6.26 & \\
\hline & 3 & $2.45 \pm 0.52$ & & \\
\hline \multirow[t]{2}{*}{ Self-confidence } & 1 & $2.72 \pm 0.93$ & & $1<3^{* *}$ \\
\hline & 2 & $2.86 \pm 0.80$ & 3.64 & \\
\hline Knowledge of career & 1 & $3.52 \pm 0.50$ & & $1>2^{*}$ \\
\hline \multirow[t]{2}{*}{ Decision making } & 2 & $3.42 \pm 0.43$ & 4.88 & $1>3^{* *}$ \\
\hline & 3 & $3.34 \pm 0.46$ & & $2>3^{*}$ \\
\hline \multirow[t]{3}{*}{ Career maturity } & 1 & $3.27 \pm 0.32$ & & $1>3^{\star *}$ \\
\hline & 2 & $3.22 \pm 0.29$ & 3.20 & \\
\hline & 3 & $3.17 \pm 0.29$ & & \\
\hline
\end{tabular}

Note: 1 = University and above; 2 = Secondary/Secondary; 3 = Primary School and below. 
Table 4. Differences in career maturity of college students with different levels of mothers education.

\begin{tabular}{|c|c|c|c|c|}
\hline Variables & $\begin{array}{l}\text { Mother's educational } \\
\text { level }\end{array}$ & $M \pm S D$ & $F$ & $L S D$ \\
\hline \multirow[t]{3}{*}{ Professional self-knowledge } & 1 & $3.73 \pm 0.58$ & 3.54 & $1>2^{\star}$ \\
\hline & 2 & $3.56 \pm 0.58$ & & $1>3^{* *}$ \\
\hline & 3 & $3.51 \pm 0.57$ & & \\
\hline Interpersonal & 1 & $3.57 \pm 0.63$ & 7.28 & $1>2^{\star *}$ \\
\hline \multirow[t]{2}{*}{ Communication strategy } & 2 & $3.28 \pm 0.68$ & & $1>3^{* * *}$ \\
\hline & 3 & $3.20 \pm 0.64$ & & \\
\hline \multirow[t]{3}{*}{ Independence } & 1 & $2.67 \pm 0.69$ & & $1>2^{*}$ \\
\hline & 2 & $2.61 \pm 0.56$ & 4.90 & $1>3^{* *}$ \\
\hline & 3 & $2.53 \pm 0.52$ & & \\
\hline \multirow[t]{3}{*}{ Feasibility } & 1 & $3.75 \pm 0.59$ & & $1<3^{*}$ \\
\hline & 2 & $3.82 \pm 0.56$ & 4.16 & $2<3^{* *}$ \\
\hline & 3 & $3.93 \pm 0.51$ & & \\
\hline \multirow[t]{3}{*}{ Utility } & 1 & $2.77 \pm 0.66$ & 3.77 & $1>2^{\star}$ \\
\hline & 2 & $2.60 \pm 0.62$ & & $1>3^{* * *}$ \\
\hline & 3 & $2.52 \pm 0.63$ & & \\
\hline \multirow[t]{3}{*}{$\begin{array}{c}\text { Knowledge of career decision } \\
\text { making }\end{array}$} & 1 & $3.57 \pm 0.40$ & & $1>2^{*}$ \\
\hline & 2 & $3.42 \pm 0.46$ & & \\
\hline & 3 & $3.40 \pm 0.42$ & 3.70 & $1>3^{*}$ \\
\hline \multirow[t]{3}{*}{ Career maturity } & 1 & $3.31 \pm 0.29$ & $\begin{array}{l}3.70 \\
3.49\end{array}$ & $1>2^{*}$ \\
\hline & 2 & $3.21 \pm 0.30$ & & $1>3^{*}$ \\
\hline & 3 & $3.20 \pm 0.30$ & & \\
\hline
\end{tabular}

Note: 1 = University and above; 2 = Secondary/Secondary; 3 = Primary School and below.

3.2. The grade of Teachers' Professional Identity of Pre-Service Kindergarten Teachers, Whether They Have Part-Time Work Experience Or Not, and the Difference of Their Parents' Education Level

\subsubsection{Grade Differences of Teachers' Professional Identity among Normal School Students in Preschool Education}

The results show that there are four dimensions of professional identity: career will and expectation, professional will, professional value. There were significant grade differences $(\mathrm{p}<0.01)$ in vocational efficacy, which were reflected in prep > grade $4>$ grade $2>$ grade $1>$ grade 3 . See Table 5 .

\subsubsection{Differences of Teachers' Professional Identity among Pre-Service Kindergarten Teachers with or without Part-Time Working Experience}

The results showed that there was a significant difference in the occupational efficacy of pre-service kindergarten teachers with or without part-time work experience $(\mathrm{p}<0.05)$. The difference is that there is a part-time and 
Table 5. Differences in grades of professional identity among normal school students majoring in preschool education.

\begin{tabular}{|c|c|c|c|c|}
\hline Variables & Grade & $M \pm S D$ & $F$ & $L S D$ \\
\hline \multirow[t]{5}{*}{ Occupational will and expectations } & 0 & $4.24 \pm 0.93$ & & $1<2^{*}$ \\
\hline & 1 & $4.14 \pm 0.92$ & & \\
\hline & 2 & $4.29 \pm 0.77$ & 1.40 & \\
\hline & 3 & $4.10 \pm 0.63$ & & \\
\hline & 4 & $4.27 \pm 0.60$ & & \\
\hline \multirow[t]{7}{*}{ Occupational will } & 0 & $2.99 \pm 0.59$ & & $0>1^{* *}$ \\
\hline & 1 & $2.74 \pm 0.63$ & & $0>2^{* * *}$ \\
\hline & 2 & $2.63 \pm 0.66$ & 8.34 & $1>3^{* *}$ \\
\hline & 3 & $2.51 \pm 0.57$ & & $1<4^{\star}$ \\
\hline & 4 & $2.95 \pm 0.56$ & & $2<1^{*}$ \\
\hline & & & & $2<4^{* *}$ \\
\hline & & & & $3<4^{* * *}$ \\
\hline \multirow[t]{5}{*}{ Occupational value } & 0 & $3.28 \pm 0.94$ & & $0>1^{\star * *}$ \\
\hline & 1 & $2.84 \pm 0.90$ & & $0>3^{* * *}$ \\
\hline & 2 & $2.73 \pm 0.90$ & 11.40 & $1>3^{* * *}$ \\
\hline & 3 & $2.36 \pm 0.82$ & & $2>3^{* *}$ \\
\hline & 4 & $2.61 \pm 0.86$ & & \\
\hline \multirow[t]{5}{*}{ Occupational performance } & 0 & $3.81 \pm 0.81$ & & $0>3^{*}$ \\
\hline & 1 & $3.70 \pm 0.85$ & & $1<3^{*}$ \\
\hline & 2 & $3.64 \pm 0.77$ & 2.53 & $3<4^{*}$ \\
\hline & 3 & $3.51 \pm 0.67$ & & \\
\hline & 4 & $3.88 \pm 0.59$ & & \\
\hline \multirow[t]{5}{*}{ Occupational identity } & 0 & $3.52 \pm 0.66$ & & $0>1^{*}$ \\
\hline & 1 & $3.35 \pm 0.61$ & & $0>3^{* * *}$ \\
\hline & 2 & $3.32 \pm 0.56$ & 4.97 & $1>3^{* *}$ \\
\hline & 3 & $3.13 \pm 0.48$ & & $2>3^{* *}$ \\
\hline & 4 & $3.42 \pm 0.46$ & & $3<4^{* *}$ \\
\hline
\end{tabular}

Note: $0=$ prep, $1=$ Grade 1, $2=$ Grade 2, $3=$ Grade 3, $4=$ Grade 4 .

professional-related < there is a part-time but not a professional-related < no-Part-time. There is no significant difference in the three dimensions of professional will and expectation, professional will and career value of preschool normal school students with or without part-time working experience. See Table 6. 
Table 6. Differences in professional identity of pre-service kindergarten teachers with or without part-time work experience.

\begin{tabular}{|c|c|c|c|c|}
\hline Variables & $\begin{array}{l}\text { Doing part-time job } \\
\text { or not }\end{array}$ & $M \pm S D$ & $F$ & $L S D$ \\
\hline \multirow[t]{3}{*}{ Occupational will and expectation } & 1 & $4.22 \pm 0.83$ & & \\
\hline & 2 & $4.17 \pm 0.83$ & 0.30 & \\
\hline & 3 & $4.19 \pm 0.80$ & & \\
\hline \multirow[t]{3}{*}{ Occupational will } & 1 & $2.73 \pm 0.64$ & & \\
\hline & 2 & $2.68 \pm 0.62$ & 0.95 & \\
\hline & 3 & $2.63 \pm 0.68$ & & \\
\hline \multirow[t]{3}{*}{ Occupational value } & 1 & $2.79 \pm 0.92$ & 0.88 & \\
\hline & 2 & $2.77 \pm 0.89$ & & \\
\hline & 3 & $2.63 \pm 0.95$ & & \\
\hline \multirow[t]{3}{*}{ Occupational performance } & 1 & $3.74 \pm 0.79$ & 4.19 & $1>2^{*}$ \\
\hline & 2 & $3.58 \pm 0.74$ & & $1>3^{*}$ \\
\hline & 3 & $3.51 \pm 0.88$ & & \\
\hline \multirow[t]{3}{*}{ Career identity } & 1 & $3.36 \pm 0.59$ & 2.37 & \\
\hline & 2 & $3.29 \pm 0.55$ & & \\
\hline & 3 & $3.22 \pm 0.60$ & & \\
\hline
\end{tabular}

Note: 1 = no part-time work; 2 = part-time but not related to major; 3 = part-time and major-related.

\subsubsection{Differences in Professional Identity of Preschool Normal Students with Different Educational Backgrounds}

The results showed that the difference of professional identity of pre-service kindergarten teachers with different father's education level was reflected in professional efficacy, specifically that the father's education level was university or above $>$ father's education level was primary school or below. The differences of professional will and expectation, professional will and professional value were not significant, and the difference of professional identity of pre-service kindergarten teachers with different mothers' education level was reflected in professional value. In particular, the mother's education level is university or above > the mother's education level is middle school/technical secondary school, primary school and below, professional will and expectation, professional will and professional efficacy. See Table 7, Table 8.

\subsection{Correlation Analysis of Variables}

The correlation analysis of controlling grade, part-time work and parents' education level showed that there was a significant positive correlation between occupational identity, learning input and Occupational maturity. In grade, there was a significant correlation between the degree of parental education and the degree of Occupational recognition and the maturity of Occupational, and the degree of father's education was significantly correlated with the level of learning input. See Table 9. 
Table 7. Differences in vocational identification among college students with father's levels.

\begin{tabular}{ccccc}
\hline Variables & Father's educational level & $M \pm S D$ & $F$ & $L S D$ \\
\hline $\begin{array}{c}\text { Occupational will and } \\
\text { expectation }\end{array}$ & 1 & $4.23 \pm 0.88$ & & \\
& 2 & $4.19 \pm 0.82$ & 0.11 & \\
Occupational will & 3 & $4.22 \pm 0.83$ & & \\
& 1 & $2.70 \pm 0.70$ & & \\
Occupational value & 2 & $2.74 \pm 0.61$ & 1.22 & \\
& 3 & $2.64 \pm 0.68$ & & \\
& 1 & $2.87 \pm 0.92$ & & \\
Occupational performance & 2 & $2.75 \pm 0.91$ & 0.65 & \\
& 3 & $2.78 \pm 0.92$ & & \\
& 1 & $3.84 \pm 0.83$ & & \\
Occupational identity & 2 & $3.67 \pm 0.78$ & 2.51 & \\
& 3 & $3.60 \pm 0.80$ & & \\
& 1 & $3.38 \pm 0.60$ & & \\
& 2 & $3.33 \pm 0.57$ & 0.37 & \\
& 3 & $3.32 \pm 0.60$ & \\
& & & & \\
& & & &
\end{tabular}

Table 8. Differences in occupational identity among college students with mother's different education levels.

\begin{tabular}{|c|c|c|c|c|}
\hline Variables & $\begin{array}{c}\text { Mother's educational } \\
\text { level }\end{array}$ & $M \pm S D$ & $F$ & $L S D$ \\
\hline \multirow[t]{3}{*}{$\begin{array}{l}\text { Occupational will and } \\
\text { expectation }\end{array}$} & 1 & $4.20 \pm 0.93$ & & \\
\hline & 2 & $4.18 \pm 0.87$ & 0.36 & \\
\hline & 3 & $4.24 \pm 0.72$ & & \\
\hline \multirow[t]{3}{*}{ Occupational will } & 1 & $2.82 \pm 0.63$ & & \\
\hline & 2 & $2.73 \pm 0.65$ & 1.89 & \\
\hline & 3 & $2.66 \pm 0.62$ & & \\
\hline \multirow[t]{3}{*}{ Occupational value } & 1 & $3.07 \pm 0.87$ & & $1>2^{*}$ \\
\hline & 2 & $2.79 \pm 0.93$ & 4.81 & $1>3^{* *}$ \\
\hline & 3 & $2.67 \pm 0.89$ & & \\
\hline \multirow[t]{3}{*}{ Occupational performance } & 1 & $3.68 \pm 0.82$ & & \\
\hline & 2 & $3.70 \pm 0.82$ & 0.25 & \\
\hline & 3 & $3.65 \pm 0.73$ & & \\
\hline \multirow[t]{3}{*}{ Occupational identity } & 1 & $3.42 \pm 0.56$ & & \\
\hline & 2 & $3.34 \pm 0.61$ & 0.72 & \\
\hline & 3 & $3.31 \pm 0.54$ & & \\
\hline
\end{tabular}

Note: 1 = University and above; 2 = Secondary/Secondary; 3 = Primary School and below. 
Table 9. Descriptive statistics and correlation analysis of variables.

\begin{tabular}{|c|c|c|c|c|c|c|c|c|c|c|}
\hline variables & $M \pm S D$ & 1 & 2 & 3 & 4 & 5 & 6 & 7 & 8 & 9 \\
\hline $\begin{array}{l}\text { 1) Occupational } \\
\text { identity }\end{array}$ & $3.34 \pm 0.58$ & 1 & & & & & & & & \\
\hline 2) Learning input & $4.11 \pm 0.87$ & $0.37^{* * *}$ & 1 & & & & & & & \\
\hline $\begin{array}{l}\text { 3) Occupational } \\
\text { decision } \\
\text { knowledge }\end{array}$ & $3.42 \pm 0.44$ & $0.30^{* * *}$ & $0.39^{* * *}$ & 1 & & & & & & \\
\hline $\begin{array}{l}\text { 4) Occupational } \\
\text { decision attitude }\end{array}$ & $3.05 \pm 0.32$ & $0.11^{\star *}$ & $0.10^{* *}$ & $0.26^{* * *}$ & 1 & & & & & \\
\hline 5) Career maturity & $3.22 \pm 0.30$ & $0.27^{* * *}$ & $0.32^{* * *}$ & $0.82^{\star * *}$ & $0.77^{* * *}$ & 1 & & & & \\
\hline 6) Grade & $1.67 \pm 1.02$ & $-0.10^{\star *}$ & -0.02 & $0.10^{\star *}$ & 0.04 & $0.09^{*}$ & 1 & & & \\
\hline $\begin{array}{l}\text { 7) Have a } \\
\text { part-time or not }\end{array}$ & $1.42 \pm 0.65$ & $-0.81^{* *}$ & -0.07 & $0.07^{*}$ & $0.10^{* *}$ & 0.16 & 0.54 & 1 & & \\
\hline $\begin{array}{l}\text { 8) Father's } \\
\text { educational level }\end{array}$ & $3.07 \pm 0.57$ & -0.03 & $-0.07^{*}$ & $-0.12^{* *}$ & -0.03 & $-0.10^{*}$ & 0.05 & 0.02 & 1 & \\
\hline $\begin{array}{l}\text { 9) Mather's } \\
\text { educational level }\end{array}$ & $3.25 \pm 0.59$ & -0.04 & 0.03 & $-0.09^{*}$ & -0.03 & $-0.07^{\star}$ & $0.01^{* *}$ & 0.05 & $0.49^{* * *}$ & 1 \\
\hline
\end{tabular}

\subsection{Occupational Identity and Occupational Rationality: Mediating Effect Test}

Occupational agrees that the relational model of learning input and Occupational maturity is shown in Figure 1.

Because of the significant correlation between the grade, part-time and parental education level and the main variables in the study, it is regarded as the controlling variable. Occupational identity is regarded as dependent variable and learning input as intermediary variable. SPSS Process component Model 4 was used for regression analysis (Hayes, 2013) [23]. Figure 1 shows that occupational identity significantly predicts learning input to significantly predict Occupational maturity Identify, learning investment significantly predict Occupational maturity. The influence of occupational identity on Occupational maturity is still significant after adding learning input, so Occupational maturity plays a part of intermediary role between Occupational identity and Occupational maturity. As the figure shows, learning commitment mediates between Occupational identity and Occupational maturity. See Table 10.

The Bootstrap method is further selected to test the confidence interval estimation. The SPSS macro model 4, which is compiled by Hayes, is used to repeat sampling 1000 times, and the $95 \%$ confidence interval is calculated. If the confidence interval obtained does not include 0 , the corresponding intermediary effect is significant. The results show that learning input plays an intermediary role in the relationship between Occupational identity and Occupational maturity. The indirect effect of learning input on Occupational maturity is significant. The confidence interval is $[0.03+0.07]$, excluding $0(\mathrm{p}<0.05)$. See Table 11 . 


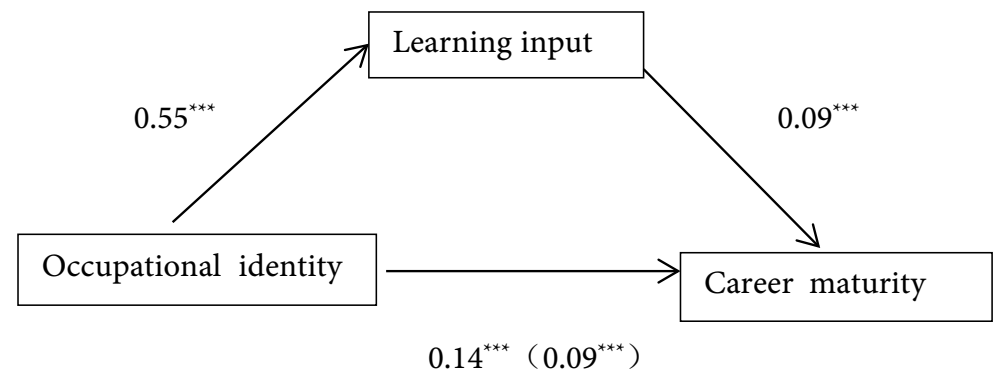

Figure 1. Intermediary model of occupational identity and occupational maturity.

Table 10. Regression analysis of variable relationships in the model.

\begin{tabular}{|c|c|c|c|c|c|c|c|c|c|}
\hline \multicolumn{2}{|c|}{ Regression equation } & \multicolumn{4}{|c|}{ Integral fitting index } & \multicolumn{4}{|c|}{$\begin{array}{l}\text { Regression coefficient } \\
\text { significance }\end{array}$} \\
\hline $\begin{array}{l}\text { Outcome } \\
\text { variable }\end{array}$ & Predictive variable & $R$ & $R-s q$ & $F$ & Coeff & Se & $t$ & $L L C I$ & $U L C I$ \\
\hline \multirow[t]{5}{*}{$\begin{array}{l}\text { Learning } \\
\text { input }\end{array}$} & Grade & 0.30 & 0.15 & 29.95 & 0.02 & 0.05 & 0.62 & -0.41 & 0.08 \\
\hline & \multicolumn{3}{|c|}{ Done part-time job or not } & & -0.61 & 0.05 & -1.26 & -0.16 & 0.03 \\
\hline & \multicolumn{3}{|c|}{ Father's educational level } & & -0.18 & 0.06 & $-2.90^{* *}$ & -0.30 & -0.06 \\
\hline & \multicolumn{3}{|c|}{ Mother's educational level } & & 0.15 & 0.06 & $2.62^{\star \star}$ & 0.04 & 0.27 \\
\hline & \multicolumn{3}{|c|}{ Occupational identity } & & 0.55 & 0.05 & $10.53^{* * *}$ & 1.83 & 2.91 \\
\hline \multirow[t]{6}{*}{$\begin{array}{l}\text { Occupational } \\
\text { maturity }\end{array}$} & Grade & 0.39 & 0.16 & 21.66 & 0.28 & 0.01 & $2.61^{*}$ & 0.07 & 0.05 \\
\hline & \multicolumn{3}{|c|}{ Done part-time jobs or not } & & 0.05 & 0.02 & $3.23^{\star \star}$ & 0.02 & 0.09 \\
\hline & \multicolumn{3}{|c|}{ Father's educational level } & & -0.02 & 0.06 & -2.90 & -0.30 & 0.02 \\
\hline & \multicolumn{3}{|c|}{ Mother's educational level } & & 0.02 & -0.04 & -1.77 & -0.08 & 0.00 \\
\hline & \multicolumn{3}{|c|}{ Learning input } & & 0.09 & 0.01 & $6.91^{\star * \star}$ & 0.06 & 0.11 \\
\hline & \multicolumn{3}{|c|}{ Occupational identity } & & 0.09 & 0.02 & $4.60^{* * *}$ & 0.05 & 0.13 \\
\hline \multirow[t]{5}{*}{$\begin{array}{l}\text { Learning } \\
\text { input }\end{array}$} & Grade & 0.31 & 0.10 & 15.43 & 0.03 & 0.01 & $2.68^{* \star}$ & 0.01 & 0.05 \\
\hline & \multicolumn{3}{|c|}{ Done part-time jobs or not } & & 0.05 & 0.02 & $2.81^{* *}$ & 0.01 & 0.08 \\
\hline & \multicolumn{3}{|c|}{ Father's educational level } & & -0.04 & 0.02 & -1.77 & -0.08 & 0.01 \\
\hline & \multicolumn{3}{|c|}{ Mother's educational level } & & -0.02 & 0.02 & -1.06 & -0.06 & 0.02 \\
\hline & \multicolumn{3}{|c|}{ Occupational identity } & & 0.14 & 0.02 & $7.44^{\star * \star}$ & 0.10 & 0.17 \\
\hline
\end{tabular}

Table 11. Model effect analysis table.

\begin{tabular}{cccccc}
\hline Routine & effect & $S e$ & $t$ & LLCI & ULCI \\
\hline Gross effect (X-Y) & 0.14 & 0.02 & $7.44^{* * *}$ & 0.10 & 0.17 \\
Direct effect (X-Y) & 0.09 & 0.02 & $4.60^{* * *}$ & 0.05 & 0.13 \\
Indigo effect (X-M-Y) & 0.05 & 0.01 & $5.76^{* * *}$ & 0.03 & 0.07 \\
\hline
\end{tabular}

Note: $\mathrm{X}=$ Occupational identity, $\mathrm{M}=$ learning input, $\mathrm{Y}=$ Occupational maturity. 


\section{Discussion}

The study shows that there are significant grade differences in career identity and maturity, which is consistent with previous studies. Through the investigation of the status quo of professional identity of normal school students by means of questionnaire and other methods, it is found that there are significant differences in professional identity among normal school students (Zang, Su Wan \& 2015) [24]. Xiong (2006) [25] made up the career maturity questionnaire of Chinese college students and studied the career maturity of 1751 college students in China. The results show that there are grade differences in the career maturity of Chinese college students. Wang (2011) [26] investigated 420 vocational students. The results showed that the vocational maturity of teachers in higher vocational colleges was significantly different. The professional maturity of senior normal students was significantly higher than that of other grades. The development of the internal dimension of professional identity of senior normal students is not balanced which may be due to the third year. The senior period is the outbreak of the crisis of vocational value of normal school students and the vocational value education is extremely urgent. Previous studies have shown that the professional identity of normal school students is closely related to their mental health, and the low level of professional identity can also lead to anxiety (Lin, Wang, Chen, Zhu, \& Wang, 2017) [27] and depression (Wang \& Zhang, 2012) [11]. Therefore, teachers' education can strengthen the students' visiting and learning, practice training and so on, so that teachers' students can explore actively and improve teachers' skills, so as to put forward some suggestions. At the same time, the students' professional efficacy and self-confidence can be promoted by propagating the outstanding deeds of the famous teachers, carrying out the face-to-face communication activities of the famous teachers, spreading the lofty teachers' professional values and guiding the normal students to set up correct values for the occupation of preschool teachers. The author should give special psychological guidance to the students in this period to relieve their psychological problems, such as anxiety and depression. The relevant departments should actively pay attention to the mental health of students, set up psychological counselling and career planning lectures, let the students have a clearer position on their own career development and relieve the psychological pressure of their students. The practice of entering kindergarten has an important influence on improving the professional maturity of preschool normal students. We can properly arrange the normal students of freshmen and sophomores to study in kindergarten, make active career exploration, and arouse their enthusiasm for self-development.

The data showed that there were significant differences in occupational identity and occupational maturity among pre-service kindergarten teachers with or without part-time working experience, and the occupational efficacy of non-part-time teachers college students was significantly higher than that of non-part-time teachers college students. The occupational maturity of teachers 
college students with part-time experience is significantly higher than that of normal students without part-time work experience. Active participation in social activities has a significant impact on the Occupational identity of normal school students (Hu \& Huang 2016) [28]. Xiong (2006) [25] thinks that the difference of Occupational maturity between practice and practice is obvious. Zheng (2006) [29] thinks that there is a significant difference in occupational maturity among pre-service kindergarten teachers with or without part-time working experience. Students without part-time jobs have no way of knowing the gap between the skills they master and the needs of their actual jobs, while students who have worked part-time know clearly their differences. Taking part-time work can make normal students recognize their shortcomings and encourage them to work harder to improve their skills. Relevant departments should encourage teachers' students to participate more with social practice, provide related practical work with major or organize related activities, deepen normal students' understanding of Occupational, let normal students learn and grow in combination of theory and practice.

The investigation showed that the normal students with father of high education had higher occupational efficacy, the normal students with mother of higher education had higher occupational value, and the normal school students with high parents' education had higher occupational maturity than those with low parents' education. Parents' good education experience can provide some valuable guidance for college students in practical sense, and provide harmonious family culture atmosphere for college students' occupational identity development psychologically and spiritually (Zhang \& Wang, 2016) [30]. Fathers have different levels of education There is a very significant difference in the development of occupational maturity of college students (Liu, 2009) [8]. The family education of preschool normal students with higher education level is more reasonable. Parents have the ability to guide or influence their children, so that the development level of occupational decision-making is obviously superior to their parents for other education. The degree of students, making their occupational maturity higher level. However, not because of the high degree of education of the father, college students' occupational maturity is high. For example, parents are less educated. Students who study or below cannot get occupational experience from their parents. They should actively collect information, seek training opportunities, and make their own occupational planning according to the actual situation, which is characterized by high initiative, independence and reality.

Studies show that occupational identity significantly in predicting Occupational maturity, which is consistent with previous study (Chen \& Li, 2015) [31]. Teachers with higher Occupational identity will be more willing to prepare for the development of occupation, study professional knowledge and skills, and actively participate in the exploration of occupational, comprehensive self-knowledge and knowledge of the occupational world, thereby enhancing its occupational maturity. Therefore, teachers, educators and related departments should strengthen 
the positive publicity of preschool education, improve the salary and social status of kindergarten workers, let students love the cause of preschool education, and guide students to set up firm occupational belief. At the same time, the school should strengthen the cooperation with the kindergarten, let the students communicate with the first-line teachers and the kindergarten directors with rich experience, and strengthen the relationship between the pre-school students and the kindergarten.

Furthermore, the role of learning input in occupational identification and occupational maturity is significant, and has been supported by relevant study (Tan \& Liu, 2014) [16]. The influence of learning investment on the maturity of occupational is mainly reflected in the promotion of students' occupational maturity by the factors of behavioural input, cognitive input and emotional input. Individuals with high level learning investment can have more positive and diligent behaviour in the process of learning and extracurricular activities, and have more cognitive methods and psychological input in learning, and experience positive emotional feelings in their study and life. In other words, they maintain a positive attitude towards learning and extracurricular activities, and show positive preparation for future occupational posts. Therefore, their overall level of occupational maturity will also be higher, learning investment and occupational maturity significantly positive correlation.

\section{The Practical Enlightenment of the Research}

Because of the particularity of preschool education, students need to learn relevant knowledge and skills specially, and the mastery of professional knowledge and skills requires continuous and dedicated learning, not only students' own efforts. Moreover, it is necessary for the school to cultivate and improve the relevant aspects. In summary, this study puts forward some suggestions for the students and the school respectively.

To the students: First, students should actively and continuously engage in learning, actively participate in all activities related to learning, not limit learning in the classroom, integrate learning into their own lives, and make learning a habit or even an ability. Let oneself not only have deep professional knowledge but also solid practical ability; Second, master scientific and reasonable learning methods, establish reasonable knowledge structure, accumulate continuously in daily study life, and lay a professional and extensive knowledge base for the future development of Occupational; Third, combining theory with practice, applying theory to practice, thus sublimating theoretical knowledge, and eventually upgrading all kinds of qualities that one has into one's own ability; Fourth, actively participate in social practice, enrich practical experience in practice, improve Occupational skills and skills, and truly prepare for the future Occupational.

To the school: first, the university should carry on the positive education to the university student, through offering the lecture psychological guidance, the optimization study environment, the establishment each scholarship and so on 
various forms, guides them to carry on the study investment positively; Second, the school encourages college students to participate in social practice, actively carry out practical activities such as the second classroom, such as going deep into kindergarten internships, conducting Occupational career interview competitions, teachers' skills and skills competitions, etc., so as to enrich practical experience. Connect with pluralistic society; third, the school should respect the students' main position, so the design and arrangement of teaching activities should first understand the actual situation, according to the needs of the development of students to formulate and implement. At the same time, we should encourage students to invest more in learning and make more efforts to prepare for the development of occupational, so as to promote the improvement of students' occupational maturity; Fourth, at the early stage of admission, the school introduces the students to the basic situation of their major, the program of professional training, the scope and direction of employment, and so on, so that students can have a preliminary occupational plan for a comprehensive understanding of the situation of professional learning. After entering the university, occupational career planning course can be set up to solve the problems of students' occupational exploration.

\section{Conclusion and Limitation of the Study}

\subsection{Conclusion}

In this study, the characteristics and influencing factors of occupational identification with occupational maturity of pre-service kindergarten teachers were analyzed through a questionnaire survey. Meanwhile, learning input was incorporated into the intermediary model of teachers' occupational identity and occupational maturity, and the relationship between them was discussed. It enriches the related theories of occupational identity and occupational maturity development of pre-school normal students. At the same time, it also emphasizes the positive role of occupational identification and learning input in promoting occupational maturity of preschool normal students. In the future intervention research, we can also pay attention to the strengthening of students' learning investment and the cultivation of occupational identity, such as create a good learning atmosphere, mobilize the enthusiasm of students, encourage students to engage in more learning, and make adequate preparation for the development of occupational; pay attention to the cultivation of teacher's occupational identity of pre-school normal school students, let students deeply realize the value of teacher's occupational and the degree of matching themselves with teacher's occupational, and have a correct understanding of preschool teacher's occupation$\mathrm{al}$, and form a proper view of occupational.

\subsection{Limitation}

However, there are still some shortcomings and limitations in this study which need to be improved in the future: First, as far as intermediary mechanism is concerned, this study shows that learning input only partially mediates the rela- 
tionship between occupational identity and occupational maturity. As this study examines the intermediate variable of learning input, study have shown that self-concept, self-supporting personality and self-efficacy are significantly associated with occupational maturity. Future research can look at variables in other areas such as self-efficacy; second, due to objective reasons, the sample ratio of this study is extremely extreme, and it is not possible to explore the gender difference of occupational maturity. The conclusion of the study needs to be tested; thirdly, the samples of this study are taken from Nanchang area of Jiangxi Province. Considering the influence of regional differences, there may be differences in the occupational maturity level of preschool education normal students in different regions, so we should be more cautious in popularizing the results. Future research needs to select larger, more representative samples; Fourth, the study adopts horizontal survey, without longitudinal study, can not reflect the dynamic relationship between the two in depth and comprehensively; Fifth, this study is only a relational study, and does not adopt an experimental study. It can't reveal the causality of teachers' occupational identity, learning investment and occupational maturity.

\section{Funding}

This work was supported by Jiangxi province university humanities and social science planning fund project "Study on the impact of pre-service kindergarten teachers' professional commitment on academic achievement and career planning", standard number: XL1412.

\section{References}

[1] Lu, N. (2016) In Recent Years Preschool Education Major Student Employment Investigation Report. Education of the Times, 2, 51-59.

[2] Xu, J.P. (2017) The Employment Situation, Problems and Countermeasures of Young Teachers and Students under the Background of Teachers' Qualification Certificates. Family Education World, 15, 44-45.

[3] Crites, J.O. and Savickas, M.L. (1996) Revision of the Career Maturity Inventory. Journal of Career Assessment, 4, 131-138. https://doi.org/10.1177/106907279600400202

[4] Wei, S.H. and Song, G.W. (2005) A Review of the Research on Teachers' Occupational Identity in Foreign Countries. Comparative Education Research, 5, 61-66.

[5] Wang, X.Q., Ceng, L.H, Zhang, D.J and Li, S. (2010) An Initial Research on the Professional Identification Scale for Normal Students. Journal of Southwest University, 36, 152-157.

[6] Crises, J.O. (1978) Theory and Research Handbook for the Career Maturity Inventory. 2nd Edition, McGraw Hill/CTB, Monterey, CA.

[7] Savickas, M.L. (1994) Measuring Career Development: Current Status and Future Directions. Career Development Quarterly, 43, 54-62. https://doi.org/10.1002/j.2161-0045.1994.tb00846.x

[8] Liu, L.M. (2009) Revision and Development Characteristics of Occupational Maturity Questionnaire for Undergraduates. Master's Thesis, Southwestern University, Chongqing. 
[9] Stanišauskienè, V. and Edintaite, G. (2016) Professional Identity Level of Social Pedagogy Students in the Context of Career Decisions. Vocational Training. Research \& Realities, 27, 42-56. https://doi.org/10.1515/vtrr-2016-0004

[10] Hou, Y.M., Hu, P.C. and Guo, S.X. (2017) Relationship between Occupational Identification and Occupational Maturity of Medical Undergraduates. Chinese Higher Medical Education, 9, 13-14.

[11] Wang, X.Q. and Zhang, D.J. (2012) The Relationship between Occupational Identity and Mental Health of Free Normal Students and Its Enlightenment. Contemporary Teacher Education, 5, 62-67.

[12] Ma, H.Y., Wei, X.Q. and Liu, S.M. (2013) Study on the Characteristics and Influencing Factors of Occupational Maturity of Free Normal College Students. Educational Research and Experiment, 1, 82-86.

[13] Connell, J.P. (1990) Context, Self, and Action: A Motivational Analysis of Self-System Processes across the Life Span. University of Chicago, Chicago.

[14] Schaufeli, W.B., Salanova, M., Gonzálezromá, V. and Bakker, A.B. (2002) The Measurement of Engagement and Burnout: A Two Sample Confirmatory Factor Analytic Approach. Journal of Happiness Studies, 3, 71-92. https://doi.org/10.1023/A:1015630930326

[15] Yang, M.M. (2017) The Relationship between Teacher's Occupational Identity and Learning Input. Journal of Pingdingshan University, 32, 119-122.

[16] Tan, X.H. and Liu, H. (2014) Relationship between Teacher Professional Identity and Study Engagement of Normal College Students. Journal of Mianyang Normal University, 33, 118-121 + 126 .

[17] Wang, Y. (2015) The Features of Teacher Professional Identity and Its Relationship with Academic Achievement and Academic Engagement among Tuition-Free Normal College Students. Research on Higher Education in Heilongjiang Province, 11, 96-100.

[18] Zhao, M. (2013) Study on the Relationship between Learning Commitment, Professional Commitment and Teacher's Occupational Maturity. Master's Thesis, Northeast Normal University, Changchun.

[19] Tian, X.X. (2016) The Relationship between Time Management Disposition and Teacher Career Maturity of Normal University Students-The Mediating Effect of Learning Engagement. Science and Education Guide Journal, 8, 66-67.

[20] Zhang, R. (2017) Research on the Influence of Learning Engagement on the Employability of College Students: Taking Academic Achievement as Mediator Variable. Master's Thesis, Shanxi University of Finance and Economics, Shanxi.

[21] Wang X.Q, Zhu J.C, Liu, L. and Chen, X.Y. (2017) Cognitive-Processing Bias in Chinese Student Teachers with Strong and Weak Professional Identity. Frontiers in Psychology, 8, Article 784.

[22] Fang, L.T., Shi, K. and Zhang, F.H. (2008) Research on Reliability and Validity of Utrecht Work Engagement Scale-Student. Chinese Journal of Clinical Psychology, 16, 618-620.

[23] Hayes, A.F. (2013) Methodology of Selective Exposure Research: Introduction to the Special Issue. Communication Methods \& Measures, 7, 145-146. https://doi.org/10.1080/19312458.2013.845500

[24] Zhang, Y.Y., Su, X. and Wan, B.Q. (2015) Current Situation and Its Influential Factors of Career Identity of Students Majored in Preschool Education. Journal of Chizhou University, 29, 10-12. 
[25] Xiong, H.X. (2006) A Study on Occupational Maturity Questionnaire for Chinese University Students and Its Characteristics. Master's Thesis, Jiangxi Normal University, Jiangxi.

[26] Wang, C.R. (2011) On the Occupational Maturity of Higher Vocational College Students and Its Relationship with Learning Input. Occupational Educational Research, 3, 17-18.

[27] Lin, S.Y., Wang, X.Q, Chen, L.W., Zhu, J.C. and Wang, Y.B. (2017) The Relationship between Student Teachers' Professional Identity and Anxiety: The Moderating Role of Campus Pressure. Open Journal of Social Sciences, 5, 214-223. https://doi.org/10.4236/jss.2017.56019

[28] Hu, W.F. and Huang, L. (2016) A Study on the Occupational Identity of Normal College Teachers and Its Influencing Factors. Educational Research and Experiment, 1, 82-86.

[29] Zheng, H.Y. (2006) The Structure and Development Characteristics of Occupational Maturity of College Students. Master's Thesis, Southwest University, Chongqing.

[30] Zhang, J. and Wang, S.C. (2016) A Study on the Influencing Factors of the Development of Occupational Identity among College Students. Modernization of Education, 3, 124-125.

[31] Chen, L.J. and Li, B.H. (2015) The Relationship between Occupational Identity and Occupational Maturity of Normal College Students and Its Enlightenment. Theory and Practice of Contemporary Education, 7, 151-155. 